\title{
COHOMOLOGIE DES VARIÉTÉS DE SIEGEL ET REPRÉSENTATIONS GALOISIENNES ASSOCIÉES AUX REPRÉSENTATIONS CUSPIDALES COHOMOLOGIQUES
}

\author{
$\operatorname{DE} G S p_{4}(\mathbb{Q})$ \\ par
}

J. Tilouine

Ce texte reprend le contenu de deux exposés qui présentent, avec commentaires (mais sans résultat nouveau), une idée de R. Taylor [11] pour construire la représentation galoisienne associée à une forme cuspidale $\pi$ sur $G S p(4)$ dont la composante à l'infini est dans la série discrète. Cette idée n'utilise pas la formule des traces pour $G S p_{4}$ mais seulement : la théorie de Hodge (et de Hodge-Tate), les relations de congruences à la Eichler-Shimura et la dualité de Poincaré. Cette méthode montre qu'on peut associer à $\pi$ une représentation galoisienne qui est, soit de la forme voulue, soit d'une forme étrange. Le théorème dans sa version finale, qui consiste à exclure la forme étrange, nécessite le recours à la formule des traces. Il est dû à Laumon [8] et à Weissauer [12]). Nous nous concentrons sur la contribution de R. Taylor, en ajoutant quelques commentaires sur les progrès de [8] et [12]. La forme finale est la suivante (les notations sont expliquées dans le texte).

Soit $G=G S p_{4}, \mathbf{A}=\mathbb{Q}_{f} \times \mathbb{Q}_{\infty}$ l'anneau des adèles rationnelles et $G(\mathbb{A})=$ $G_{f} \times G_{\infty}$, avec $G_{f}=G S p_{4}\left(\mathbb{Q}_{f}\right)$ et $G_{\infty}=G S p_{4}(\mathbb{R})$. Soit $T$ le tore de $G$ des $t=\operatorname{diag}\left(t_{1}, t_{2}, \nu t_{2}^{-1}, \nu t_{1}^{-1}\right)$. Soit $\lambda: t \mapsto t_{1}^{a} t_{2}^{b}(a \geq b \geq 0)$ un poids dominant pour $(G, B, T)$; soit $\hat{\lambda}$ le poids dominant dual et $\Pi_{\widehat{\lambda}+\rho}$ le paquet des séries discrètes de $G_{\infty}$ de caractère infinitésimal $\widehat{\lambda}+\rho$ ( $\rho$ demi-somme des racines positives). Ce paquet comprend deux éléments $\pi_{\hat{\lambda}+\rho}^{H}$ et $\pi_{\hat{\lambda}+\rho}^{W}$.

Théorème 0.1. - Soit $\pi_{f}$ une représentation lisse admissible de $G_{f}$ pour laquelle il existe des représentations lisses admissibles de $G_{f}, \pi_{1}$ et $\pi_{2}$, équivalentes à $\pi_{f}$ en presque toute place, et telles que $\pi_{1} \otimes \pi_{\widehat{\lambda}+\rho}^{H}$ et $\pi_{2} \otimes \pi_{\grave{\lambda}+\rho}^{W}$ soient automorphes cuspidales.

Alors, pour tout nombre premier $\ell$, il existe une représentation galoisienne continue 


$$
\rho_{\pi, \ell}: \operatorname{Gal}(\overline{\mathbb{Q}} / \mathbb{Q}) \rightarrow G L_{4}\left(\overline{\mathbb{Q}}_{\ell}\right)
$$

non-ramifiée hors de $\operatorname{Ram}(\pi) \cup\{\ell, \infty\}$ et telle que pour tout nombre premier $q$ en-dehors de cet ensemble fini,

$$
\operatorname{det}\left(1_{4}-X \cdot \rho_{\pi, \ell}\left(F r_{q}\right)\right)=P_{\pi, q}^{*}\left(q^{a+b+3} X\right),
$$

où $P_{\pi, q}^{*}\left(q^{-s}\right)^{-1}$ est le facteur d'Euler en $q$ de la fonction $L$ automorphe de degré 4 associée à $\pi$.

On définit ainsi un système strictement compatible de représentations galoisiennes, pures de poids $a+b+3$. Ces représentations sont de Hodge-Tate de poids $0, b+1, a+2, a+b+3$.

La précaution concernant $\pi_{1}$ et $\pi_{2}$ au lieu de $\pi_{f}$ vient de ce que la multiplicité un pour $\pi_{f} \otimes \pi_{\infty}^{H}$, conjecturée, n'est pas établie. Elle le sera dès que le transfert global de $G S p(4)$ à $G L_{4}$ sera établi [1].

Nous concluons ce texte par une description conjecturale de toutes les représentations galoisiennes associées à une représentation cuspidale cohomologique de $G S p(4, \mathbb{Q}$ ) (mais dont la composante à l'infini n'est pas nécessairement dans la série discrète). Dans cette partie, nous suivons et développons légèrement l'approche suivie par T. Itô dans la dernière section de [7].

\section{Variétés de Siegel-Shimura, systèmes locaux}

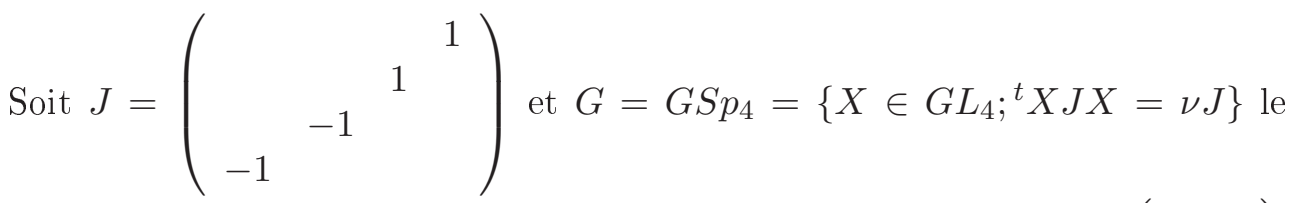
schéma en groupes réductifs déployés sur $\mathbb{Z}$ associé à $J$. On note $s=\left(\begin{array}{ll}0 & 1 \\ 1 & 0\end{array}\right)$ et $1_{2}=\left(\begin{array}{ll}1 & \\ & 1\end{array}\right)$.

On introduit le diagramme des sous-groupes paraboliques standard

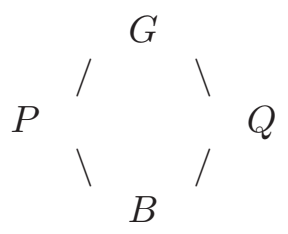

où $B$ désigne le sous-groupe de Borel constitué des matrices triangulaires supérieures de $G, P$ désigne le groupe des matrices triangulaires supérieures par blocs $(1,2,1)$ de $G$, et $Q$ celui des matrices triangulaires par blocs $(2,2)$ de $G$. On introduit les décompositions de Levi $Q=M U$ et $B=T N$, où $M$ est 
isomorphe à $G L(2) \times \mathbb{G}_{m} \operatorname{par}(A, \nu) \mapsto \operatorname{diag}\left(A, \nu s^{t} A s\right)$, et $T$ est le tore de rang 3 des $t=\operatorname{diag}\left(t_{1},, t_{2}, \nu t_{2}^{-1}, \nu t_{1}^{-1}\right)$. $U$ consiste en les matrices $\left(\begin{array}{cc}1_{2} & u \\ 0 & 1_{2}\end{array}\right)$ où $u=\left(\begin{array}{ll}a & b \\ c & a\end{array}\right)$.

Soit $\mathbf{A}=\mathbb{Q}_{f} \times \mathbb{Q}_{\infty}$ l'anneau des adèles rationnelles; on note $G_{\mathbf{A}}=G_{f} \times G_{\infty}$ la décomposition du groupe des A-points de $G$.

Soit $h: \operatorname{Res}_{\mathbb{C} / \mathbb{R}} \mathbb{C}^{\times} \rightarrow G_{/ \mathbb{R}}$ le morphisme envoyant $x+i y \operatorname{sur}\left(\begin{array}{cc}x 1_{2} & y s \\ -y s & x 1_{2}\end{array}\right)$

Soit $X$ l'orbite de $h$ par conjugaison sous $G_{\infty}$ et $C_{\infty}=\operatorname{Stab}_{G_{\infty}}(h)$.

Soit $K \subset G_{f}$ un sous-groupe compact ouvert. La variété de Siegel-Shimura (de genre 2) de niveau $K$ est définie comme

$$
S_{K}=G_{\mathbb{Q}} \backslash G_{\mathbf{A}} / K C_{\infty}=G_{\mathbb{Q}} \backslash\left(X \times G_{f} / K\right)
$$

Si $K$ est net (le sous-groupe de $\mathbb{C}^{\times}$engendré par les valeurs propres des éléments de $K G_{\infty} \cap G_{\mathbb{Q}}$ ne contient pas de racine de l'unité autre que 1 ), $S_{K}$ est une variété analytique complexe lisse de dimension 3 , et aussi une variété algébrique complexe quasi-projective lisse (non projective). Si $K^{\prime} \subset K$, on a un morphisme fini $S_{K^{\prime}} \rightarrow S_{K}$. Si $g \in G_{f}$, la multiplication à droite $r_{g}: G_{\mathbf{A}} \rightarrow G_{\mathbf{A}}$ par $\mathrm{g}$ induit une correspondance algébrique $T_{K}(g)$ (dont les deux projections sont finies)

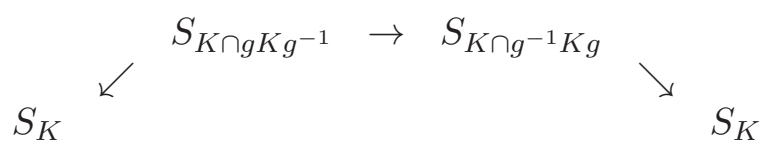

On munit ces variétés de systèmes locaux comme suit. Soit $X^{*}(T)$ le groupe des caractères de $T$. On paramétrise ce groupe par le réseau $L$ de $\mathbb{Z}^{3}$ des triplets $(a, b ; c)$ tels que $c \equiv a+b(\bmod 2) ; L \rightarrow X^{*}(T),(a, b ; c) \mapsto\left(t \mapsto t_{1}^{a} t_{2}^{b} \nu^{\frac{c-a-b}{2}}\right)$. La restriction de ce caractère au centre est $z \mapsto z^{c}$. On prend $c=a+b$ dans la suite.

Soit $\lambda \in X^{*}(T)$ dominant pour $(G, B, T)$; on a donc $a \geq b \geq 0$. 'Soit $V_{\lambda}$ la représentation de Weyl de $G$ de plus haut poids $\lambda$. On définit le système local $V_{\lambda, K}$ sur $S_{K}$ comme le faisceau des sections localement constantes du fibré $G_{\mathbb{Q}} \backslash\left(G_{\mathbf{A}} \times V_{\lambda}\right) / K C_{\infty} \rightarrow G_{\mathbb{Q}} \backslash G_{\mathbf{A}} / K C_{\infty}$, l'action sur $G_{\mathbf{A}} \times V_{\lambda}$ étant donnée par $\gamma(g, v) k c_{\infty}=\left(\gamma g k c_{\infty}, \gamma v\right)$.

Les faisceaux $V_{\lambda, K}$ sont compatibles aux morphismes $S_{K^{\prime}} \rightarrow S_{K}$.

En faisant agir la multiplication à droite par $g \in G_{f}$ trivialement sur $V_{\lambda}$, on définit une action de la correspondance algébrique $T_{K}(g) \operatorname{sur}\left(S_{K}, V_{\lambda, K}\right)$. 


\section{Cohomologie}

Pour tout $g_{f} \in G_{f}$ et tout compact ouvert $K \subset G_{f}$, on a donc une action de $T_{K}(g)$ sur $H^{\bullet}\left(S_{K}, V_{\lambda, K}\right)$. Ces actions sont compatibles aux morphismes de transition $S_{K^{\prime}} \rightarrow S_{K}$. En passant à la limite inductive on obtient une action de $G_{f}$ sur

$$
H^{\bullet}\left(S, V_{\lambda}\right)=\operatorname{indlim}_{K} H^{\bullet}\left(S_{K}, V_{\lambda, K}\right)
$$

C'est un $G_{f}$-module admissible, i.e. les $K$-invariants sont de dimension finie : $H^{\bullet}\left(S, V_{\lambda}\right)^{K}=H^{\bullet}\left(S_{K}, V_{\lambda, K}\right)$. Par le théorème de comparaison de de Rham, et en tirant les faisceaux par $G_{\mathbf{A}} \rightarrow S=G_{\mathbb{Q}} \backslash\left(G_{\mathbf{A}} / C_{\infty}\right)$

$$
\left.H^{\bullet}\left(S, V_{\lambda}\right)=H^{\bullet}\left(\mathcal{E}^{\bullet}\left(S, V_{\lambda}\right)\right)\right)=H^{\bullet}\left(\mathcal{E}^{\bullet}\left(G_{\mathbf{A}}, V_{\lambda}\right)^{G_{\mathbb{Q}}-e q u i v, C_{\infty}-i n v .}\right)
$$

Grâce à l'isomorphisme $G_{\mathbf{A}} \times V_{\lambda} \rightarrow G_{\mathbf{A}} \times V_{\lambda},(g, v) \mapsto\left(g, g_{\infty}^{-1} v\right)$, on a

$$
H^{\bullet}\left(\mathcal{E}^{\bullet}\left(G_{\mathbf{A}}, V_{\lambda}\right)^{G_{\mathbb{Q}}-e q u i v, C_{\infty}-i n v .}\right)=H^{\bullet}\left(\mathcal{E}^{\bullet}\left(G_{\mathbf{A}}, V_{\lambda}\right)^{G_{\mathbb{Q}}-i n v, C_{\infty}-e q u i v}\right)
$$

et on reconnaît dans le membre de droite la cohomologie d'algèbre de Lie relative, qu'on calcule par le complexe de Koszul relatif. On peut donc exprimer le membre de droite en termes de groupes de $\left(\mathfrak{g}, C_{\infty}\right)$-cohomologie :

$$
\left.H^{\bullet}\left(\mathcal{E}^{\bullet}\left(G_{\mathbf{A}}, V_{\lambda}\right)\right)^{G_{\mathbb{Q}}-i n v, C_{\infty}-e q u i v}\right)=H^{\bullet}\left(\mathfrak{g}, C_{\infty}, \mathcal{C}^{\infty}\left(G_{\mathbb{Q}} \backslash G_{\mathbf{A}}\right) \otimes V_{\lambda}\right) .
$$

Ceci permet d'introduire en plus de $H_{c}^{\bullet}(S, V)$ et $H_{!}^{\bullet}=\operatorname{Im}\left(H_{c}^{\bullet} \rightarrow H^{\bullet}\right)$ la cohomologie $L^{2}$ et la cohomologie cuspidale.

On définit des inclusions

$$
\mathcal{C}_{\text {cusp }}^{\infty} \subset \mathcal{C}_{c / \text { centre }}^{\infty} \subset \mathcal{C}_{(2)}^{\infty} \subset \mathcal{C}^{\infty}
$$

où la condition de cuspidalité est définie par $\int_{\mathcal{N}_{\mathbb{Q}} \backslash \mathcal{N}_{\mathbf{A}}} f(g n) d n=0, \mathcal{N}$ désignant le radical unipotent d'un des paraboliques $P, Q, B$. La première inclusion est donnée par un système inductif de troncatures lisses par des compacts mod. centre de plus en plus grands, les autres inclusions étant naturelles. Les $\left(\mathfrak{g}, C_{\infty}\right)$-modules correspondent définissent les cohomolologies cuspidales, à support compact, $L^{2}$, resp. classique. Ces inclusions induisent des morphismes

$$
H_{\text {cusp }}^{\bullet}\left(S, V_{\lambda}\right) \rightarrow H_{c}^{\bullet}\left(S, V_{\lambda}\right) \rightarrow H_{(2)}^{\bullet}\left(S, V_{\lambda}\right) \rightarrow H^{\bullet}\left(S, V_{\lambda}\right)
$$

Rappelons que Borel a démontré que l"application induite

$$
H_{\text {cusp }}^{\bullet}\left(S, V_{\lambda}\right) \rightarrow H_{!}^{\bullet}\left(S, V_{\lambda}\right)
$$

est injective. 


\section{Cohomologie cuspidale}

On rappelle que le module de Harish-Chandra $\left(\mathcal{C}_{\text {cusp }}^{\infty}\right)^{\left(C_{\infty}\right)}$ de $\mathcal{C}_{\text {cusp }}^{\infty}$ est le sous- $\left(\mathfrak{g}, C_{\infty}\right.$ )-module (admissible) constitué des vecteurs $C_{\infty}$-finis (dont la $C_{\infty^{-}}$ orbite engendre un sous-espace de dimension finie).

On démontre (livre de Borel-Wallach) que l'inclusion $\left(\mathcal{C}_{\text {cusp }}^{\infty}\right)^{\left(C_{\infty}\right)} \otimes V_{\lambda} \subset \mathcal{C}_{\text {cusp }}^{\infty} \otimes$ $V_{\lambda}$ de $\left(\mathfrak{g}, C_{\infty}\right)$-modules induit un isomorphisme de $\left(\mathfrak{g}, C_{\infty}\right)$-cohomologie. On peut décomposer le $\left(\mathfrak{g}, C_{\infty}\right)$-module de Harish-Chandra de $\mathcal{C}_{\text {cusp }}^{\infty}$ en constituants simples:

$$
\mathcal{C}_{\text {cusp }}^{\infty}\left(G_{\mathbb{Q}} \backslash G_{\mathbf{A}}\right)^{\left(C_{\infty}\right)}=\bigoplus_{\pi \text { cusp. }} m_{\pi} \cdot \pi_{f} \otimes \pi_{\infty}^{\left(C_{\infty}\right)}
$$

où $\pi=\pi_{f} \otimes \pi_{\infty}$ parcourt les classes d'isomorphisme de représentations cuspidales, $m_{\pi}$ est la multiplicité de $\pi$ et $\pi_{\infty}^{\left(C_{\infty}\right)}$ désigne le $\left(\mathfrak{g}, C_{\infty}\right)$-module des vecteurs lisses de $\pi_{\infty}$. On a de plus la décomposition

$$
H_{\text {cusp }}^{\bullet}\left(S, V_{\lambda}\right)=\bigoplus_{\pi \text { cusp. }} m_{\pi} \cdot \pi_{f} \otimes H^{\bullet}\left(\mathfrak{g}, C_{\infty}, \pi_{\infty}^{\left(C_{\infty}\right)} \otimes V_{\lambda}\right)
$$

Pour chaque $\pi=\pi_{f} \otimes \pi_{\infty}$ cuspidale, la $\left(\mathfrak{g}, C_{\infty}\right)$-cohomologie de $\pi_{\infty}^{\left(C_{\infty}\right)} \otimes V_{\lambda}$ est nulle à moins que le caractère infinitésimal du module coefficient ne soit trivial ; ceci équivaut à dire que le caractère infinitésimal de $\pi_{\infty}$ est égal à celui de $V_{\lambda}^{\vee}=V_{\widehat{\lambda}}$, la représentation de Weyl de plus haut poids $\hat{\lambda}=(a, b ;-c)$. De plus on a la décomposition de Hodge :

$$
H^{m}\left(\mathfrak{g}, C_{\infty}, \pi_{\infty}^{\left(C_{\infty}\right)} \otimes V_{\lambda}\right)=\bigoplus_{u+v=m} H^{u, v}\left(\mathfrak{g}, \mathfrak{q}, \pi_{\infty}^{\left(C_{\infty}\right)} \otimes V_{\lambda}\right)
$$

(voir [2] ou [6]).

Soit $\mathfrak{t}$ l'algèbre de Lie réelle du tore $T$; notons que par différentiation, $X^{*}(T)$ se plonge canoniquement comme réseau dans le dual linéaire de $\mathfrak{t}$. 'Soit $\rho=$ $(2,1 ; 0)$ la demi-somme des racines positives de $(G, B, T)$. Par l'isomorphisme d'Harish-Chandra

$$
\gamma: Z \mathfrak{g} \cong\left(\operatorname{Sym}^{\bullet} \mathfrak{t}\right)^{W}
$$

on peut interpréter le caractère infinitésimal de $V_{\widehat{\lambda}}$ comme $\widehat{\lambda}+\rho$. La somme ne porte donc que sur les $\pi$ tels que $\chi_{\pi_{\infty}}=\widehat{\lambda}+\rho$. La classification de VoganZuckerman pour $G=G S p_{4}$ donne les modules de Harish-Chandra avec cette propriété :

- La série discrète contient deux telles représentations : $\pi_{\hat{\lambda}+\rho}^{H}$ et $\pi_{\widehat{\lambda}+\rho}^{W}$; elles sont telles que

-la $(\mathfrak{g}, \mathfrak{q})$-cohomologie de $\pi_{\widehat{\lambda}+\rho}^{H} \otimes V_{\lambda}$ est nulle sauf en bidegré $(3,0)$ et $(0,3)$, où elle est de dimension 1 , 
-la $(\mathfrak{g}, \mathfrak{q})$-cohomologie de $\pi_{\hat{\lambda}+\rho}^{W} \otimes V_{\lambda}$ est nulle sauf en bidegré $(2,1)$ et $(1,2)$, où elle est de dimension 1 ,

Si $a>b>0$, il n'y a pas d'autre représentations.

- Si $a>0, b=0$, il y a une représentation $\pi_{\lambda}^{1}$ unitaire mais pas tempérée; elle n'est donc pas dans la série discrète ; elle contribue aux $H^{2,0}$ et $H^{0,2}$ (chacun est de dimension 1), et aux $H^{3,1}$ et $H^{1,3}$ (chacun de dimension 1).

- Si $a=b>0$, il y a deux représentations $\pi_{\lambda}^{2, \pm}$, avec $\pi_{\lambda}^{2,-}=\pi_{\lambda}^{2,+} \otimes s g n$. Elles sont également non tempérées. Elle interviennent dans $H^{1,1}$ et $H^{2,2}$ (à chaque fois avec dimension 1 )

- Si $a=b=0$, les représentations sont $\nu^{c / 2}$ et $\nu^{c / 2} \otimes s g n$; elles interviennent dans $H^{0,0}, H^{1,1}, H^{2,2}$ et $H^{3,3}$ (à chaque fois avec dimension 1 ).

On définit la composante $\pi_{f}$-isotypique du $G_{f}$-module $H_{\text {cusp }}^{\bullet}\left(S, V_{\lambda}\right)$ comme $H_{\text {cusp }}^{\bullet}\left[\pi_{f}\right]=\pi_{f} \otimes W_{\pi_{f}}$ où $W_{\pi_{f}}=\operatorname{Hom}_{G_{f}}\left(\pi_{f}, H_{\text {cusp }}^{\bullet}\right)$.

$$
H_{\text {cusp }}^{\bullet}=\bigoplus_{\pi \text { cusp. }} H_{\text {cusp }}^{\bullet}\left[\pi_{f}\right]
$$

Notons que par la classification de VZ, on peut estimer la dimension (finie) de $W_{\pi_{f}}$ :

$$
W_{\pi_{f}}=\bigoplus_{\pi=\pi_{f} \otimes \pi_{\infty} \text { cusp. }} m_{\pi} \cdot H^{\bullet}\left(\mathfrak{g}, C_{\infty}, \pi_{\infty}^{\left(C_{\infty}\right)} \otimes V_{\lambda}\right)
$$

donc

$\operatorname{dim} W_{\pi_{f}}=2 m\left(\pi_{f} \otimes \pi_{\widehat{\lambda}+\rho}^{H}\right)+2 m\left(\pi_{f} \otimes \pi_{\widehat{\lambda}+\rho}^{W}\right)+4 m\left(\pi_{f} \otimes \pi_{\lambda}^{1}\right)+2 m\left(\pi_{f} \otimes \pi_{\lambda}^{2, \pm}\right)$

où $\pi_{\lambda}^{1}$ resp. $\pi_{\lambda}^{2, \pm}$ peut intervenir en poids $(a, 0)$ resp. $(a, a)$.

\section{Cohomologie $\ell$-adique}

On va introduire les trois ingrédients-clés pour l'étude des représentations galoisiennes :

-décomposition de Hodge-Tate et endomorphisme de Sen,

-dualité de Poincaré

-relations d'Eichler-Shimura.

Les $S_{K}$ admettent des modèles canoniques sur $\operatorname{Spec} \mathbb{Q}$. Plus précisément, pour chaque sous-groupe compact ouvert $K$, soit $\mathbb{Q}_{K}$ le sous-corps de $\mathbb{Q}^{a b}$ fixé par $[\nu(K), \mathbb{Q}]$. Il y a des modèles $S_{K} / \mathbb{Q}$ munis de morphismes $S_{K} \rightarrow \operatorname{Spec} \mathbb{Q}_{K}$ à fibres géométriques connexes, qui sont espaces de modules fins de variétés abéliennes principalement polarisées (en abrégé, VAPP) avec structure de niveau $K$; on note $A_{K}$ la VAPP universelle $A_{K} \rightarrow S_{K}$ définies sur $\mathbb{Q}_{K}$. 
Les morphismes de transition $S_{K^{\prime}} \rightarrow S_{K}$ sont compatibles à l'inclusion $\mathbb{Q}_{K} \subset$ $\mathbb{Q}_{K^{\prime}}$.

Soit $\bar{x}$ un point géométrique de $S_{K}$, on a une représentation continue

$$
\phi_{\bar{x}}: \pi_{1}\left(S_{K}, \bar{x}\right) \rightarrow G S p\left(T_{\ell} A_{K, \bar{x}}\right)=G S p_{4}\left(\mathbb{Z}_{\ell}\right)
$$

4.1. Décomposition de Hodge-Tate et endomorphisme de Sen. Soit $\lambda$ un poids dominant pour $(G, B, T)$. La composée de $\phi_{\bar{x}}$ avec l'action de $G\left(\mathbb{Z}_{\ell}\right)$ sur $V_{\lambda}\left(\mathbb{Q}_{\ell}\right)$ fournit un faisceau lisse sur le modèle canonique $S_{K}$. En passant à la limite sur les niveaux, on obtient un $G_{f} \times \operatorname{Gal}(\overline{\mathbb{Q}} / \mathbb{Q})$-module

$$
H_{\mathrm{et}}^{\bullet}\left(S \otimes \overline{\mathbb{Q}}, V_{\lambda}\left(\mathbb{Q}_{\ell}\right)\right)
$$

Par Faltings-Chai Chap.VI, il est de Hodge-Tate. Pour décrire sa décomposition de Hodge-Tate, on introduit le sous-ensemble $W^{M}$ du groupe de Weyl $W$ constitué des representants de Kostant de $W_{M} \backslash W$. Ils sont définis par la condition $w\left(\Phi_{G}^{+}\right) \cap \Phi_{G}^{-} \subset \Phi^{M-}$. Soit $s_{\alpha}$ resp. $s_{\beta}$ la réflexion associée à la racine courte $\alpha=(1,-1 ; 0)$, resp. à la racine longue $\beta=(2,0 ; 0)$. On voit facilement que $W^{M}=\left\{w_{0}, w_{1}, w_{2}, w_{3}\right\}$ où $w_{0}=I d, w_{1}=s_{\beta}, w_{2}=s_{\beta} s_{\alpha}, w_{3}=s_{\beta} s_{\alpha} s_{\beta}$. Posons $H_{w_{0}}=0, H_{w_{1}}=-b-1, H_{w_{2}}=-a-2$ et $H_{w_{3}}=-a-b-3$.

Suivant Faltings-Chai, on définit des $G_{f}$-modules admissibles $A_{w_{u}}^{v}(\lambda) 0 \leq$ $u, v \leq 3$ en termes de la cohomologie cohérente du complexe BGG dual; par exemple, $A_{w_{3}}^{0}(\lambda)=\operatorname{indlim}_{K, \Sigma} H^{0}\left(\bar{S}_{K}^{\Sigma}, \omega^{a+3, b+3}\right)$, où $\bar{S}_{K}^{\Sigma}$ désigne la compactification toroidale de $S_{K}$ associée à l'éventail $\Sigma$ et $\omega^{i, j}(i \geq j \geq 0)$ est le prolongement canonique à $\bar{S}_{K}^{\Sigma}$ du fibré des différentielles relatives de $A_{K} / S_{K}$ de poids $(i, j)$. Rappelons que le faisceau $\omega^{i, j}$ sur $S_{K}$ est le faisceau localement libre de rang $i-j+1$ associé à la représentation $\operatorname{Sym}^{i-j} \otimes \operatorname{det}^{j} \mathrm{St}_{2}$ du sous-groupe de Levi $M$ de $Q$. On a alors la décomposition de Hodge-Tate $G_{f} \times \operatorname{Gal}(\overline{\mathbb{Q}} / \mathbb{Q})$-équivariante :

$$
H_{\mathrm{et}}^{m}\left(S \otimes \overline{\mathbb{Q}}, V_{\lambda}\left(\mathbb{Q}_{\ell}\right)\right) \otimes \hat{\overline{\mathbb{Q}}}_{\ell}=\bigoplus_{u+v=m} A_{w_{u}}^{v}(\lambda) \otimes \hat{\overline{\mathbb{Q}}}_{\ell}\left(H_{w_{u}}(\lambda)\right)
$$

On abrège $H_{w_{i}}(\lambda)$ en $H_{i}$.

Soit $W_{\pi, \ell}^{3}=\operatorname{Hom}_{G_{f}}\left(\pi_{f}, H_{\mathrm{et}}^{3}\left(S \otimes \overline{\mathbb{Q}}, V_{\lambda}\left(\overline{\mathbb{Q}}_{\ell}\right)\right)\right)$. Posons $m^{H}\left(\pi_{f}\right)=m\left(\pi_{f} \otimes \pi_{\bar{\lambda}+\rho}^{H}\right)$ et $m^{W}\left(\pi_{f}\right)=m\left(\pi_{f} \otimes \pi_{\widehat{\lambda}+\rho}^{W}\right)$

Le corollaire suivant est une application directe s'un théorème de Sen :

Corollaire 4.1. - Soit $\pi$ cuspidale telle que $m^{H}(\pi)>0$ et $m^{W}(\pi)>0$. Considérons la représentation galoisienne $\mathrm{Gal}\left(\overline{\mathbb{Q}}_{\ell} / \mathbb{Q}_{\ell}\right) \rightarrow G L\left(W_{\pi, \ell}^{3}\right)$. Soit $C_{\ell}$ son image et $\mathfrak{c}_{\ell}$ son algèbre de Lie. Il existe un endomorphisme $\Phi$ de $\mathfrak{c}_{\ell} \otimes \widehat{\overline{\mathbb{Q}}}_{\ell}$ de valeurs propres $H_{i} i=0,1,2,3$, avec multiplicité resp. $m^{H}\left(\pi_{f}\right), m^{W}\left(\pi_{f}\right)$, $m^{W}\left(\pi_{f}\right), m^{H}\left(\pi_{f}\right)$. 
Démonstration : Cela résulte immédiatement du théorème de Sen suivant. Soit $\left(\sigma_{\ell}, V_{\ell}\right)$ une représentation $\ell$-adique de Hodge-Tate de $\operatorname{Gal}\left(\overline{\mathbb{Q}}_{\ell} / \mathbb{Q}_{\ell}\right)$, soit $\mathfrak{g}_{\ell}$ l'algèbre de Lie de $\operatorname{Im} \sigma_{\ell}$; Il existe alors un endomorphisme $\Phi \in \mathfrak{g}_{\ell}\left(\widehat{\overline{\mathbb{Q}}}_{\ell}\right)$ dont les valeurs propres sur $V_{\ell} \otimes \otimes \widehat{\overline{\mathbb{Q}}}_{\ell}$ sont les poids de Hodge-Tate comptés avec multiplicités. Pour déterminer les multiplicités, on vérifie que pour $u+v=3$, la dimension de $\operatorname{Hom}_{G_{f}}\left(\pi_{f}, A_{w_{u}}^{v}(\lambda)\right)$ est égale à $m^{H}\left(\pi_{f}\right)$ si $u v=0$, et à $m^{W}\left(\pi_{f}\right)$ si $u$ ou $v$ vaut 2. Pour cela, on utilise un théorème de M. Harris [6] qui décompose le $G_{f}$-module $A_{w_{u}}^{v}(\lambda)$ (donné par une limite inductive d'espaces de cohomologie cohérente) comme somme des

$$
\pi_{f} \otimes H^{u, v}\left(\mathfrak{g}, \mathfrak{q}, \pi_{\infty} \otimes V_{\lambda}\right)^{\oplus m(\pi)}
$$

Ces derniers espaces ont la dimension voulue grâce à la classification de VZ rappelée ci-dessus. Il reste à rappeler que les espaces $A_{w_{u}}^{v}(\lambda)$ ont une structure $\mathbb{Q}$-rationnelle naturelle puisque ce sont des espaces de cohomologie cohérente de faisceaux définis sur $\mathbb{Q}$; donc les $\operatorname{Hom}_{G_{f}}\left(\pi_{f}, A_{w_{u}}^{v}(\lambda)\right)$ ont une structure $\overline{\mathbb{Q}}$ rationnelle naturelle, de sorte que les dimensions sur $\mathbb{C}$ ou sur $\widehat{\overline{\mathbb{Q}}}_{\ell}$ des gradués sont les mêmes.

4.2. Dualité de Poincaré. — Le cup-produit induit un accouplement parfait (sur les $K$-invariants, pour chaque $K$ net)

$H_{\mathrm{et}, !}^{m}\left(S \otimes \overline{\mathbb{Q}}, V_{\lambda}\left(\mathbb{Q}_{\ell}\right)\right) \times H_{\mathrm{et}, !}^{6-m}\left(S \otimes \overline{\mathbb{Q}}, V_{\widehat{\lambda}}\left(\mathbb{Q}_{\ell}\right)\right) \rightarrow H_{\mathrm{et}, \mathrm{c}}^{6}\left(S \otimes \overline{\mathbb{Q}}, V_{(0,0 ; c)}\left(\mathbb{Q}_{\ell}\right)\right) \stackrel{t r}{\rightarrow} \mathbb{Q}_{\ell}(-3-c)$

Cet accouplement est $G_{f} \times \operatorname{Gal}(\overline{\mathbb{Q}} / \mathbb{Q})$-équivariant si l'on munit $\mathbb{Q}_{\ell}(-3-c)$ de l'action de $G_{f}$ par $g_{f} \mapsto\left\|\nu\left(g_{f}\right)\right\|^{-c}$.

On omet le caractère algébrique $\nu$ dans la formule qui suit :

Lemme 4.2. -

$$
H_{\mathrm{et}, \mathrm{c}}^{6}\left(S \otimes \overline{\mathbb{Q}}, V_{(0,0 ; c)}\left(\overline{\mathbb{Q}}_{\ell}\right)\right)=\bigoplus_{\chi}\left(\chi\|\cdot\|^{-c}\right) \otimes\left(\chi^{\mathrm{gal}} \chi_{\ell}^{-3-c}\right)
$$

où $\chi: \mathbb{Q}_{f}^{\times} / \mathbb{Q}^{\times+} \rightarrow \overline{\mathbb{Q}}_{\ell}^{\times}$parcourt les caractères continus d'image finie et $\chi_{\ell}$ est le caractère cyclotomique $\ell$-adique. Dans cette décomposition, la trace est le morphisme de projection sur le caractère trivial.

Du point de vue galoisien, on a en effet par dualité de Poincaré étale :

$$
\left.H_{\mathrm{et}, \mathrm{c}}^{6}\left(S \otimes \overline{\mathbb{Q}}, V_{(0,0 ; c)}\left(\overline{\mathbb{Q}}_{\ell}\right)\right)\right)=H_{\mathrm{et}}^{0}\left(\operatorname{Spec}\left(\mathbb{Q}^{\mathrm{ab}}\right) \otimes \overline{\mathbb{Q}}, V_{(0,0 ;-3-c)}\left(\overline{\mathbb{Q}}_{\ell}\right)\right)=\bigoplus_{\chi}\left(\chi^{\mathrm{gal}} \chi_{\ell}^{-3-c}\right)
$$

L'identification du $H^{0}$ de $S$ avec celui de $\operatorname{Spec}\left(\mathbb{Q}^{\text {ab }}\right)$ vient de la théorie des modèles canoniques rappelée ci-dessus. La partie concernant $G_{f}$ vient de la fonctorialité de cette dualité pour les correspondances algébriques et de ce que 
la théorie des modèles canoniques précise que dans l'identification du $H^{0}$ de $S$ avec celui de $\operatorname{Spec}\left(\mathbb{Q}^{\text {ab }}\right)$, l'action de $g_{f} \in G_{f}$ se fait par la loi d'Artin de $\nu\left(g_{f}\right)$. Rappelons que si $\pi$ est cuspidale sur $G(\mathbf{A})$, de caractère central $\omega_{\pi}$ (d'ordre fini), on a $\pi_{\mathfrak{q}} \cong \pi_{q}^{*} \otimes \omega_{\pi, q}$ en tout $q$ non-ramifé. En effet, le paramètre de Langlands local en une telle place est

$$
\sigma\left(\pi_{q}\right)\left(F r_{q}\right)=\left(\begin{array}{cccc}
\alpha_{q} & & & \\
& \beta_{q} & & \\
& & \gamma_{q} & \\
& & & \delta_{q}
\end{array}\right)
$$

(où $\left.P_{\pi, q}^{*}(X)=\left(1-\alpha_{q} X\right)\left(1-\beta_{q} X\right)\left(1-\gamma_{q} X\right)\left(1-\delta_{q} X\right)\right)$ et

$$
\sigma\left(\omega_{\pi, q}\right)=\nu \circ \sigma\left(\pi_{q}\right): F r_{q} \mapsto \alpha_{q} \delta_{q}=\beta_{q} \gamma_{q},
$$

donc

$$
\sigma\left(\pi_{q}\right) \cong \sigma\left(\pi_{q}\right)^{*} \otimes \sigma\left(\omega_{\pi, q}\right)
$$

R. Taylor pose dans [11] la question : a t-on globalement $\pi \cong \pi^{*} \otimes \omega_{\pi}$ ? Nous remercions le rapporteur qui a attiré notre attention sur le fait suivant : la réponse à cette question est positive.

En effet, pour toute place $v$ de $\mathbb{Q}$, la représentation locale $\pi_{v}$ satisfait $\pi_{v} \cong$ $\pi_{v}^{*} \otimes \omega\left(\pi_{v}\right)$ puisque $g \mapsto \pi_{v}\left({ }^{t} g^{-1}\right)$ réalise la dualité et que $g J^{t} g=\lambda(g) J$.

En l'absence de réponse à cette question au moment de la rédaction de [11], Taylor a eu recours à une astuce : introduire un ensemble fini $\Pi$ de représentations cuspidales $\pi$ presqu'équivalentes, c.à.d. dont les paramètres locaux $\sigma\left(\pi_{q}\right)$ sont égaux pour presque tout $q$, et qui est stable par $\pi \mapsto \pi^{*} \otimes \omega_{\pi}$. Nous appelerons un tel ensemble $\Pi$ un paquet autodual tordu. Pour un tel paquet, soit $W_{\Pi, \ell}^{i}=\bigoplus_{\pi \in \Pi} W_{\pi, \ell}^{i}$ et $W_{\Pi}=\bigoplus_{i} W_{\Pi, \ell}^{i}$.

Il résulte de la dualité de Poincaré que

$$
W_{\Pi, \ell}^{i} \cong\left(W_{\Pi, \ell}^{6-i}\right)^{*} \otimes \overline{\mathbb{Q}}_{\ell}\left(\omega_{\pi}^{\text {gal }} \chi_{\ell}^{-3-c}\right)
$$

( $\omega_{\pi}^{\text {gal }}$ ne dépend pas de $\pi \in \Pi$ par Cebotarev).

4.3. Relations d'Eichler-Shimura. - Soit $K$ un compact ouvert net. Soit $q$ un premier ne divisant pas le niveau de $K$; soit $\mathcal{H}_{G, q}$ resp. $\mathcal{H}_{M, q}$, la $\mathbb{Q}$-algèbre de Hecke non ramifiée de $G\left(\mathbb{Q}_{q}\right)$ resp. de $M\left(\mathbb{Q}_{q}\right)$. Soit $\widetilde{S}_{G}^{M}: \mathcal{H}_{G, q} \rightarrow \mathcal{H}_{M, q}$ la transformée de Satake partielle tordue $\widetilde{S}_{G}^{M}(f)(m)=\int_{U\left(\mathbb{Q}_{q}\right)} f(m u) d u$. L'algèbre $\mathcal{H}_{M, q}$ est engendrée sur

$\mathcal{H}_{G, q}$ par $U_{q}=M\left(\mathbb{Z}_{q}\right) \operatorname{diag}(1,1, q, q) \cdot M\left(\mathbb{Z}_{q}\right)$. Le polynôme minimal $P_{q}(X)$ de $U_{q}$ sur $\mathcal{H}_{G, q}$ est un polynôme unitaire de degré 4 appelé polynôme de Hecke. Pour toute représentation cuspidale $\pi$ cohomologique telle que $\pi^{K} \neq 0$, soit $\lambda_{\pi}$ : $\mathcal{H}_{G, q} \rightarrow \overline{\mathbb{Q}}$ la représentation donnant l'action de $\mathcal{H}_{G, q}$ sur la droite $\pi_{q}^{G\left(\mathbb{Z}_{q}\right)}$. Soit 
$P_{\pi, q}(X)$ le polynôme réciproque de $P_{\pi, q}^{*}(X)=\left(1-\alpha_{q} X\right) \ldots$ La spécialisation de $P_{q}(X)$ via $\lambda_{\pi}$ est $q^{4(c+3)} P_{\pi, q}\left(q^{-c-3} X\right)$.

Faltings-Chai ont montré ([3] Chap.VI) que

Proposition 4.3. - (Relations d'Eichler-Shimura) Le Frobenius géométrique $F r_{q}$ agissant sur $H_{e t, *}^{\bullet}\left(S_{K} \otimes \overline{\mathbb{Q}}, V_{\lambda}\left(\mathbb{Q}_{\ell}\right)\right.$ ) est annulé par $P_{q}(X)$ (avec $*=\emptyset$ ou c).

Si on prend $c=a+b$ comme dans le Théorème, comme les représentations cuspidales sont dans la cohomologie intérieure (qui est pure), on a

Corollaire 4.4. - Le Frobenius géométrique $F r_{q}$ agissant sur $W_{\pi, \ell}^{\bullet}$ est annulé par $q^{4(a+b+3)} P_{\pi, q}\left(q^{-a-b-3} X\right)$. Les valeurs propres de $q^{a+b+3} \sigma\left(\pi_{q}\right)$ ont pour module $q^{a+b+3}$.

Dans cette deuxième partie, on présente, à des fins pédagogiques mais sans apport nouveau, certains aspects de la construction de la représentation galoisienne associée à une forme cuspidale $\pi$ sur $G S p(4)$; on étudie d'abord le cas où la composante à l'infini est dans la série discrète, puis on décrit sans démonstration les autres cas (on donne les résultats complets, non tous démontrés à ce jour). On suit une approche de R. Taylor [11] qui n'utilise pas la formule des traces pour $G S p_{4}$ mais seulement : la théorie de Hodge-Tate, les relations d'Eichler-Shimura et la dualité de Poincaré. Cette méthode montre qu'on peut associer à $\pi$ de type à l'infini dans la série discrète, une représentation galoisienne qui est, soit de la forme voulue, soit d'une forme étrange. Dans le cas où $\pi_{\infty}$ est dans la série discrète, seul le recours à la formule des traces [8], [12], permet d'exclure cette forme.

\section{La méthode de Taylor}

Soit $\pi$ cuspidale telle que $m^{H}(\pi)>0$ ou $m^{W}(\pi)>0$. Soit $\Pi$ un paquet autodual tordu (cf. Sect. 4.2 de CVS), c'est un ensemble fini de représentations cuspidales contenant $\pi$ et stable par dualité tordue. Soit $E$ un corps $\ell$-adique sur lequel sont définies toutes les parties finies $\pi_{f}$ de $\Pi$. Soit $N(\Pi)$ un multiple commun des niveaux des $\pi \in \Pi$. On forme la semi-simplification $W$ du $E[\operatorname{Gal}(\overline{\mathbb{Q}} / \mathbb{Q})]$-module $W_{\Pi, \ell}^{3}$. Par hypothèse, $W \neq 0$. Soit $H$ l'enveloppe de Zariski de l'image de Galois dans $G L_{E}(W)$. Ce groupe algébrique est réductif comme enveloppe de Zariski d'un groupe agissant sur une somme d'irréductibles. Soit $s: H \hookrightarrow G L_{E}(W)$ la représentation fidèle de $H$ sur $W$ et $r: \operatorname{Gal}(\overline{\mathbb{Q}} / \mathbb{Q}) \rightarrow H(E)$ la représentation galoisienne correspondante.

Par dualité de Poincaré, on a un accouplement parfait

$$
\langle\bullet, \bullet\rangle: W \times W \rightarrow E\left(\omega_{\pi}^{\text {gal }} \chi_{\ell}^{-3-c}\right)
$$


Il existe un caractère algébrique $n: H \rightarrow \mathbb{G}_{m}$ défini sur $E$ tel que $n \circ r=$ $\omega_{\pi}^{\text {gal }} \chi_{\ell}^{-3-c}$ et on a pour tout $h \in H:\left\langle h w, h w^{\prime}\right\rangle=n(h)\left\langle w, w^{\prime}\right\rangle$.

Il s'ensuit que les valeurs propres des $h \in H$ interviennent par paires $\{\alpha, n(h) / \alpha\}$. Par les relations de congruence, on va voir en fait que :

Lemme 5.1. - Si $h \in H$, ses valeurs propres sont contenues dans l'union d'au plus deux paires $\{\alpha, n(h) / \alpha\}$.

En effet, pour $h$ dans la classe de conjugaison de $r\left(F r_{q}\right)(q \neq \ell$ premier à $N(\Pi)$ ), cela résulte des relations de congruences d'Eichler-Shimura. On voit facilement que cette condition est Zariski-fermée.

Soit $H^{0}$ la composante neutre de $H$ et $T_{H} \subset B_{H} \subset H^{0} \otimes \overline{\mathbb{Q}}_{\ell}$ un tore déployé maximal et un sous-groupe de Borel.

Corollaire 5.2. - La représentation algébrique $W \otimes \overline{\mathbb{Q}}_{\ell}$ fidèle de $H^{0}$ est telle que :

1) les poids de $\left(H^{0}, B_{H}, T_{H}\right)$ sont de la forme $\left(\lambda_{1}, \lambda_{2}, n / \lambda_{1}, n / \lambda_{2}\right)$ et les multiplicités de $\lambda_{i}$ et $n / \lambda_{i}$ sont égales.

2) Il y a un élément $\phi \in T_{H}(\bar{E})$ tel que mult $\lambda_{1}(\phi)=m^{H}(\Pi)$ et mult $\lambda_{2}(\phi)=$ $m^{W}(\Pi)$.

3) $\operatorname{dim} W^{\lambda_{1}}=\operatorname{dim} W^{n / \lambda_{1}}=m^{H}(\Pi)$ et $\operatorname{dim} W^{\lambda_{2}}=\operatorname{dim} W^{n / \lambda_{2}}=m^{W}(\Pi)$.

En effet, soient $\lambda_{1}, \ldots, \lambda_{n}$ les poids de $H^{0}$ sur $W$. Soit $t \in T_{H}(\bar{E})$ un élément suffisamment régulier : $\lambda_{i}(t) \neq \lambda_{j}(t)$ pour tout $i \neq j$ et $\lambda_{i}(t) \lambda_{j}(t) \neq n(t)$ si $\lambda_{i} \lambda_{j} \neq n$.

Par le lemme la liste est en fait $\lambda_{1}, \lambda_{2}, n / \lambda_{1}, n / \lambda_{2}$; par l'accouplement de dualité, mult $\left(\lambda_{i}\right)=\operatorname{mult}\left(n / \lambda_{i}\right)$.

Par le théorème de Sen, on a $\Phi \in \mathfrak{c}_{\ell} \subset$ Lie $H^{0} \otimes \bar{E}$ de valeurs propres $H_{i}$ $(=0,1,2,3)$ sur $W$ (on peut supposer qu'il est défini sur $\bar{E}$ au lieu de $\widehat{\bar{E}})$. On prend alors $\phi=\exp \ell^{2} \Phi$. Les valeurs propres de $\phi$ sont donc $\lambda_{1}(\phi)=\exp \ell^{2} H_{0}$, $\lambda_{2}(\phi)=\exp \ell^{2} H_{1},\left(n / \lambda_{2}\right)(\phi)=\exp \ell^{2} H_{2}$, et $\left(n / \lambda_{1}\right)(\phi)=\exp \ell^{2} H_{3}$.

Pour conclure concernant les multiplicités, il ne reste plus qu'à vérifier que $\lambda_{i} \neq n / \lambda_{i}$. Si l'égalité avait lieu, en prenant $h=\phi$, on trouverait $2 H_{i}=-c-3$ et donc $H_{i}=H_{3-i}$, ce qui est faux car ces poids sont deux à deux distincts. En particulier, les multiplicités des poids ont les valeurs annoncées.

On en déduit que le groupe semisimple $\left(H^{0}\right)^{\prime}$ est de rang au plus deux; la classification des groupes semisimples de rang $\leq 2$ sur un corps algébriquement clos de caractéristique zéro fournit la liste de possibilités pour les couples $\left(\left(H^{0}\right)^{\prime},\left.s\right|_{\left(H^{0}\right)^{\prime}}\right)$, puis pour $\left.s\right|_{H^{0}}$ ainsi que les groupes (finis) d'automorphismes extérieurs de $H^{0}$ préservant cette représentation (pages 298-299 de [11]).

En utilisant alors un

Lemme 5.3. - (Serre) 
Soit $F$ un corps de nombres et $E$ un corps $\ell$-adique. Soit $H$ un groupe algébrique défini sur $E$ et $R: \operatorname{Gal}(\bar{F} / F) \rightarrow H(E)$ continue d'image Zariski dense. Soit $V$ un fermé de Zariski de $H$ stable par conjugaison et de dimension strictement plus petite que celle de $H$. Alors l'ensemble des places $v$ de $F$ telles que $R\left(F r_{v}\right) \in V(E)$ est de densité nulle.

Taylor obtient plusieurs théorèmes.

5.1. Cas $m^{H}(\pi)>0$ et $m^{W}(\pi)>0$. - L'hypothèse que $m^{H}(\Pi)>0$ et $m^{W}(\Pi)>0$ permet d'exclure certains cas. Il reste :

Théorème 5.4. - Soit $\pi_{f}$ une représentation lisse admissible de $G_{f}$. Supposons qu'il existe deux représentations lisses admissibles $\pi_{1}$ et $\pi_{2}$ de $G_{f}$ presqu'équivalentes à $\pi_{f}$, telles que $\pi_{1} \otimes \pi_{\widehat{\lambda}+\rho}^{H}$ et $\pi_{1} \otimes \pi_{\widehat{\lambda}+\rho}^{W}$ soient cuspidales pour $G(\mathbf{A})$. On a l'un des cas suivants :

1) Il existe $R: \operatorname{Gal}(\overline{\mathbb{Q}} / \mathbb{Q}) \rightarrow G L_{4}\left(E^{\prime}\right)$ (pour une extension finie $E^{\prime}$ de $E$ ) non-ramifiée hors de $\operatorname{Ram} \pi \cup\{\ell, \infty\}$, et telle que pour pour tout premier hors d'un ensemble de densité nulle, $R\left(F r_{q}\right)$ a le polynôme caractéristique voulu. et

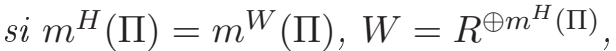

2) cas endoscopique : il existe $R_{i}: \operatorname{Gal}(\overline{\mathbb{Q}} / \mathbb{Q}) \rightarrow G L_{2}\left(E^{\prime}\right)(i=1,2)$ non ramifées hors de $\operatorname{Ram} \pi \cup \operatorname{Ram} \pi_{1} \cup \operatorname{Ram} \pi_{2} \cup\{\ell, \infty\}$, telles que hors d'un ensemble de densité nulle de premiers q, le polynôme caractéristique de $R_{1} \oplus$ $R_{2}\left(F r_{q}\right)$ est le polynôme voulu. De plus, $W=R_{1}^{m^{H}(\Pi)} \oplus R_{2}^{m^{W}(\Pi)}$.

3) cas potentiellement $C A P:$ il y a une extension finie galoisienne de groupe sur $\mathbb{Q}$ contenu dans $D_{8}$, et deux caractères continus $\chi_{i}: \operatorname{Gal}(\overline{\mathbb{Q}} / K) \rightarrow$ $\left(E^{\prime}\right)^{\times}\left(i=1,2\right.$ tels que $\left.W\right|_{K}$ (après restriction $\grave{a} K$ ) est isomorphe $\grave{a}$ $\left(\left.\chi_{1} \oplus\left(\omega_{\pi}^{\text {gal }} \chi_{\ell}^{-3}\right)\right|_{K} \chi_{1}^{-1}\right)^{m^{H}(\Pi)} \oplus\left(\left.\chi_{2} \oplus\left(\omega_{\pi}^{\text {gal }} \chi_{\ell}^{-3}\right)\right|_{K} \chi_{2}^{-1}\right)^{m^{W}(\Pi)}$

Le cas 3) est ETRANGE; il ne doit pas intervenir dans la série discrète. mais ne peut être exclu par l'analyse de R. Taylor. De plus dans les cas 1) et 2), il y a a priori un ensemble exceptionnel de densité zéro de premiers non ramifiés où l'on ne connaît pas le polynôme caractéristique de Frobenius.

Le théorème est complété par [8] et [12].

On obtient le Th. annoncé. On conjecture que dans le cas 1), la représentation est irréductible. C'est par exemple le cas si $\pi$ est le lifting de Yoshida d'une forme cuspidale de Hilbert sur un corps quadratique réel.

Le cas 2) s'appelle endoscopique, on l'obtient de la manière suivante : on fixe deux entiers $u>v \geq 2$ avec $u \equiv v(\bmod 2)$; on prend une forme cuspidale classique $f$, resp. $g$, de poids $u$, resp. de poids $v$ le lifting de Yoshida associé à $f \times g$ fournit une forme cuspidale de Siegel dont la représentation galoisienne de degré 4 est

$$
R=\rho_{f, \ell} \oplus \rho_{g, \ell} \chi_{\ell}^{u-v} \quad \text { et } W=\rho_{f, \ell}^{m^{H}(\pi)} \oplus\left(\rho_{g, \ell} \chi_{\ell}^{u-v}\right)^{m^{W}(\pi)} .
$$


Remarque : Noter que les cas 1$)$ et 2), il résulte du Théorème de FaltingsChai Chap.VI sur les poids de la cohomologie $H_{\text {et,! }}^{3}\left(S \otimes \overline{\mathbb{Q}}, V_{\lambda}\left(\mathbb{Q}_{\ell}\right)\right)$ que les représentations $R$ de degré quatre sont pures de poids $w=k_{1}+k_{2}-3$.

5.2. Cas où $m^{1}(\pi)+m^{2}(\pi)>0$. - On obtient les représentations de type CAP. Taylor obtient aussi des théorèmes dans ces cas. Dans la section cidessous, on voit comment elles entrent dans le paysage.

\section{Panorama complet conjectural}

On trouve dans [7] une interprétation de [1] donnant la classification des représentations galoisiennes associées aux formes cuspidales pour $G S p_{4}$. Cette classification sera complètement démontrée lorsque le transfert de $G S p_{4}$ à $G L_{4}$ sera établi (par Arthur).

La mention (R), resp. (NR) signifie que $\pi$ est tempérée, ou de manière équivalente, que $\pi$ satisfait la conjecture de Ramanujan : $\left|\alpha_{i}\right|=p^{\frac{w}{2}}$ avec $w=a+b+3$ pour toute valeur propre $\alpha_{i}$ du Frobenius $\rho_{\pi, \ell}\left(F r_{q}\right), q \neq \ell, q \notin \operatorname{Ram}(\pi)$.

Les représentations galoisiennes associées à une représentation automorphe dans la série discrète de $L^{2}\left(G_{\mathbb{Q}} \backslash G_{\mathbb{A}}\right)$ sont de l'un des six types suivants. Les cinq premiers types sont cuspidaux, le dernier ne l'est pas.

1. représentation "générale" $(\mathrm{R})$

On a $\Pi=\left\{\pi_{f}\right\}, m^{H}(\pi)=m^{W}(\pi)>0$ et $\rho_{\pi, \ell}$ est irréductible de dimension 4 d'image contenue dans $G S p_{4}$, pure de poids $a+b+3$, son $D_{\mathrm{HT}}$ (covariant) intervient dans $H^{w, 0} \oplus H^{a+2, b+1} \oplus H^{b+1, a+2} \oplus H^{0, w}=$ $\operatorname{grH}_{d R, !}^{3}\left(S, \mathcal{V}_{\lambda}\right)$

2. représentation "de type Yoshida" $(\mathrm{R})$

C'est le cas endoscopique. On a $\Pi=\left\{\pi_{f}\right\}, m^{H}(\pi)>0, m^{W}(\pi)>0$ et

$$
\rho_{\pi, \ell}=\rho_{f, \ell} \oplus \rho_{g, \ell} \chi_{\ell}^{-\frac{u-v}{2}}
$$

où les deux représentations de dimension 2 sont irréductibles $(f, g$ cuspidales elliptiques) En fait, $u=w+1, v=a-b+2$ et $\frac{u-v}{2}=b+1$ et $\pi=\Theta(f \times g)$ est le Theta lift de H. Yoshida (Inv. Math.1980).

L'image de $\rho_{\pi, \ell}$ est contenue dans $\left(G L_{2} \times G L_{2}\right)^{0} \subset G S p_{4}$. De plus, $D_{\mathrm{HT}}\left(\rho_{f, \ell}\right)$ intervient dans $H^{w, 0} \oplus H^{0, w}$ et $D_{\mathrm{HT}}\left(\rho_{g, \ell} \chi_{\ell}^{-\frac{u-v}{2}}\right)$ dans $H^{a+2, b+1} \oplus H^{b+1, a+2}$ tous ces termes sont des sous-quotients de $\left.H_{d R, !}^{3}\left(S, \mathcal{V}_{\lambda}\right)\right)$. Il s'ensuit

$$
W_{\Pi, \ell}^{\bullet}=\rho_{f, \ell}^{m^{H}(\Pi)} \oplus\left(\rho_{g, \ell} \chi_{\ell}^{-\frac{u-v}{2}}\right)^{m^{W}(\Pi)}
$$

3. représentation "de type Soudry" (NR) 
cas où les $\pi \in \Pi$ sont Cuspidales Associées au parabolique de Klingen (CAP Klingen), ceci suppose $\lambda=(a, 0)$. On a $m^{1}(\Pi)>0$ et $m^{2}(\Pi)=$ $m^{H}(\Pi)=m^{W}(\Pi)=0$

$$
\rho_{\pi, \ell}=\rho_{f, \ell} \oplus \rho_{f, \ell} \chi_{\ell}^{-1}
$$

Pour mieux évoquer l'intervention de l'opérateur de Lefschetz qui explique la théorie de Hodge de cette représentation, on notera

$$
\rho_{\pi, \ell}=\rho_{f, \ell} \oplus \rho_{f, \ell}(-1)
$$

Son image est contenue dans le Levi du parabolique de Siegel de $G S p_{4}$. De plus, $\rho_{f, \ell}$ irréductble pure de poids $w-1$ ( $f$ forme cuspidale elliptique de poids $w) D_{\mathrm{HT}}\left(\rho_{f, \ell}\right)$ intervient dans $H^{w-1,0} \oplus H^{0, w-1}$ qui provient de $H_{d R, !}^{2}$ (contribution de $m\left(\pi_{f} \otimes \pi_{\lambda}^{1}\right)>0$ ) et $D_{\mathrm{HT}}\left(\rho_{f, \ell}(-1)\right)$ est pur de poids $w+1$ intervient dans $H^{w, 1} \oplus H^{1, w}$, (dans la décomposition de $H_{d R, !}^{4}$ ). On a $W_{\Pi, \ell}^{\bullet}=\rho_{\pi, \ell}^{m^{1}(\Pi)}$.

4. représentation "de type Saito-Kurokawa" (NR)

Cas des Cuspidales Associées au parabolique de Siegel (CAP Siegel), ceci suppose $\lambda=(a, a)$. C'est le cas où $m^{2}(\Pi)>0$. On a alors $m^{1}(\Pi)=0$ et $m^{H}(\Pi)>0$ ou $m^{W}(\Pi)>0$. Blasius-Rogawski (BR) ont conjecturé qu'alors $m^{H}(\Pi)>0$ et $m^{W}(\Pi)=0$. On a

$$
\rho_{\pi, \ell}=\chi \oplus \rho_{f, \ell} \oplus \chi(-1)
$$

Son image est contenue dans le Levi du parabolique de Siegel de $G S p_{4}$. De plus, $\rho_{f, \ell}$ est irréductible, pure de poids $w$, associée à une forme cuspidale elliptique de poids $w+1$. En admettant la conjecture de BR, on voit que $D_{\mathrm{HT}}\left(\rho_{f, \ell}\right)$ intervient dans $H^{w, 0} \oplus H^{0, w}$ donc dans $H_{d R, !}^{3}$.

Le caractère $\chi$ est de la forme $\chi=\chi_{\ell}^{-a-1} \epsilon$ ( $\epsilon$ d'ordre fini) et son image $D_{\mathrm{HT}}(\chi)$ intervient dans $H^{a+1, a+1}$, un sous-quotient de $H_{d R, !}^{2}$, et celle de $\chi(-1)$ intervient dans $H^{a+2, a+2}$, sous-quotient de $H_{d R, !}^{4}$; donc

$$
W_{\Pi, \ell}^{\bullet}=\chi^{A} \oplus \rho_{f, \ell}^{B} \oplus \chi(-1)^{A}
$$

5. représentation "de type Howe, Piatetskii-Shapiro" (NR)

Cuspidale Associée au Borel (CAP Borel) C'est le cas où $m^{2}(\Pi)>0$, $m^{1}(\Pi)=0$ et $m^{H}(\Pi)=m^{W}(\Pi)=0$.

On a

$$
\rho_{\pi, \ell}=\chi_{1} \oplus \chi_{2} \oplus \chi_{1}(-1) \oplus \chi_{2}(-1)
$$

Son image est contenue dans le tore maximal standard de $G S p_{4}$. les caractères $\chi_{i}$ interviennent dans $H^{a+1, a+1}$ dans le $H_{!}^{2}$, et leurs twists par 
-1 interviennent dans le $H_{!}^{4}$. et

$$
W_{\Pi, \ell}^{\bullet}=\chi_{1}^{A} \oplus \chi_{2}^{B} \oplus \chi_{1}(-1)^{A} \oplus \chi_{2}(-1)^{B}
$$

pour certains $A, B \geq 1$.

6. représentation "de type dimension 1 "

C'est un cas dans la série discrète non cuspidale. $\rho_{\pi, \ell}=\chi \oplus \chi(-1) \oplus$ $\chi(-2) \oplus \chi(-3)$.

Pour conclure, on peut noter que par [4], la correspondance locale de Langlands pour $G S p_{4}$ est maintenant complète de sorte que la question de la question de sa compatibilité à la correspondance globale ci-dessus peut être posée avec précision. Les résultats connus concernent la compatibilité dans le cas des séries principales non-ramifiées, certaines séries modérément ramifiiée (par A. Genestier et l'auteur) et le cas de la représentation de Steinberg, par A. Genestier. Les autres cas de niveau Iwahorique sont tous décrits conjecturalement dans [10].

\section{Références}

[1] J. Arthur :, Automorphic Forms of GSp(4), in Contributions to Automorphic Forms, Geometry, and Number Theory, pp.65-82, a volume in honor of J. Shalika, ed. by H. Hida, D. Ramakrishnan and F. Shahidi, Johns Hopkins University Press 2004.

[2] A. Borel, N. Wallach : Continuous cohomology, discrete subgroups and representations of reductive groups, 2nd edition, Amer. Math. Soc., Providence, RI, 2000.

[3] G. Faltings, C.-L. Chai : Degeneration of abelian varieties, Erg. der Math. u. ihre Grenzgebiete, Springer Verlag 1990.

[4] W.-T. Gan, S. Takeda : The local Langlands correspondence for GSp(4), ArXiv :0706.0952v3[mathNT].

[5] A. Genestier, J. Tilouine :Systèmes de Taylor-Wiles pour GSp(4), in "Formes Automorphes (II), le cas du groupe GSp(4)", pp.177-290, Astérisque 302, 2005.

[6] M. Harris : Automorphic forms of $\bar{\partial}$-cohomology type as coherent cohomology classes, J. Diff. Geom. 32 (1990), 1-63.

[7] T. Itô : On motives and $\ell$-adic Galois representations associated to automorphic representations on GSp(4), in On Automorphic Forms on the Symplectic Similitude Group GSp(4). Proc. 9th Autumn Workshop in Hakuba, edited by M. Furusawa, 2007.

[8] G. Laumon : Fonctions zêtas des variétés de Siegel de dimension trois, Formes Automorphes (II), le cas du groupe GSp(4), pp. 151-176, Astérisque 302, SMF, 2005.

[9] A. Mokrane, J. Tilouine : Cohomology of Siegel varieties with p-adic integral coefficients and applications, pp.1-95 (2002), Astérisque 280, Publ. Soc. Math. France.

[10] B. Roberts, R. Schmidt : Local Newforms for GSp(4), Springer Lecture Notes in Mathematics Vol 1918 (2007), 312 pages. 
[11] R. Taylor : On the cohomology of Siegel threefolds, Inv. Math.114 (1993), pp.289310.

[12] R. Weissauer : Four-dimensional Galois representations, Formes Automorphes (II), le cas du groupe GSp(4), pp. 151-176, Astérisque 302, SMF, 2005.

7 avril 2008

J. Tilouine, LAGA, Institut Galilée, Université Paris 13, 93430 Villetaneuse E-mail : tilouine@math.univ-paris13.fr 